\title{
$O$ perfil das tecnologias em saúde incorporadas no SUS de 2012 a 2019: quem são os principais demandantes?
}

\author{
The profile of health technologies incorporated in SUS during 2012- \\ 2019: who are the mainly demanders?
}

Francisco José Rodrigues Filho', Michelly Cristiny Pereira ${ }^{\mathbf{2}}$

DOI: 10.1590/0103-1104202113011

RESUMO As tecnologias em saúde têm revolucionado a assistência médica e a gestão em saúde. A Comissão Nacional de Incorporação de Tecnologias no SUS (Conitec) é o órgão do Ministério da Saúde que assessora na incorporação, exclusão ou alteração de novas tecnologias no Sistema Único de Saúde (SUS). O presente estudo objetivou descrever o perfil das tecnologias incorporadas no SUS de 1 de janeiro de 2012 a 30 de setembro de 2019. Os dados foram coletados no site da Conitec. Na análise estatística, foi utilizado o teste Qui-quadrado de Pearson e o Teste Exato de Fisher. Os resultados demonstram que foram incorporadas 380 tecnologias, prevalecendo os medicamentos (46,6\%). Em relação aos demandantes, os de origem interna superaram os demais (82,4\%), principalmente secretarias do Ministério da Saúde ( $\mathrm{p}<0,001)$. As Doenças Infecciosas e Parasitárias (DIPs) foram as mais beneficiadas (20,3\%), com destaque para o HIV (Vírus da Imunodeficiência Humana). A maioria das tecnologias incorporadas passou por consulta pública $(\mathrm{p}<0,001)$. Conclui-se que o perfil das tecnologias incorporadas são principalmente medicamentos, por demanda interna, com indicação para DIPs e, sobretudo para o HIV. Os medicamentos continuam sendo o foco das solicitações e as demandas internas passaram a ter mais espaço nesse cenário.

PALAVRAS-CHAVE Sistema Único de Saúde. Tecnologia biomédica. Avaliação da tecnologia biomédica.

1 Hospital das Clínicas da Universidade Federal de Pernambuco - Recife (PE), Brasil.

2 Universidade Federal de Pernambuco (UFPE) Recife (PE), Brasil. michelly.pereira@ufpe.br
ABSTRACT Health technologies have revolutionized medical care and health management. The National Commission for the Incorporation of Technologies in the SUS (Conitec) is the Ministry of Health's body that advises on the incorporation, exclusion or alteration of new technologies in the Unified Health System (SUS). This study aimed to describe the profile of technologies incorporated in the SUS between January 1, 2012 and September 30, 2019. Data were collected on the Conitec website. Statistical analysis used Pearson's chi-square test and Fisher's exact test. The results show that 380 technologies were incorporated, with medication prevailing (46.6\%). In relation to the plaintiffs, those of internal origin surpassed the others (82.4\%), mainly secretariats of the Ministry of Health $(p<0.001)$. Infectious and Parasitic Diseases (PIDs) were the most benefited (20.3\%), with emphasis on HIV (Human Immunodeficiency Virus). Most of the incorporated technologies underwent public consultation $(p<0.001)$. It is concluded that the profile of the incorporated technologies are mainly medicines, by internal demand, with indication for PIDs and, above all, for HIV. Medicines continue to be the focus of requests and internal demands have gained more space in this scenario.

KEYWORDS Unified Health Systems. Biomedical technology. Technology assessment, biomedical. 


\section{Introdução}

A Constituição Federal Brasileira, de 1988, reconheceu a saúde como um direito de todos e um dever do Estado, forçando-o a garantir acesso universal e equitativo a medicamentos e outras tecnologias em saúde' Tecnologias em saúde são medicamentos, procedimentos, produtos e protocolos utilizados no atendimento ao paciente ${ }^{2}$.

A incorporação de tecnologias no Sistema Único de Saúde (SUS) foi normatizada, pela primeira vez, em 2006, através das portarias $\mathrm{n}^{0} 152$ e $\mathrm{n}^{0} 3.323$. Nessa época, o fluxo se dava através da articulação entre Secretaria de Atenção à Saúde (SAS), Secretaria de Ciência, Tecnologia e Insumos Estratégicos (SCTIE), Secretaria de Vigilância em Saúde (SVS), Agência Nacional de Saúde Suplementar (ANS) e Agência Nacional de Vigilância Sanitária (Anvisa) ${ }^{3}$.

Ainda segundo a Conitec ${ }^{3}$, cabia à SAS a gestão do processo de incorporação de tecnologias e à SCTIE a gestão da avaliação das tecnologias de interesse para o SUS. Foi instituída, sob a coordenação da SAS, a Comissão para Incorporação de Tecnologias do Ministério da Saúde (Citec), com a missão de encaminhar o processo de admissibilidade de tecnologias em consonância com as necessidades sociais em saúde e de gestão do SUS. Em 2008, a coordenação da Citec foi transferida para a SCTIE ${ }^{3}$.

Em 2011, a Citec foi ampliada e intitulada Comissão Nacional de Incorporação de Tecnologias no SUS (Conitec), através da Lei $n^{0} 12.401 / 2011$, que alterou a Lei no 8.080/1990 (Lei Orgânica do SUS). É um órgão da estrutura regulatória do Ministério da Saúde, que assessora na incorporação, exclusão ou alteração de novas tecnologias em saúde no SUS4.

$\mathrm{O}$ arcabouço de funcionamento da Conitec se dá por dois fóruns: O Plenário da Conitec e a Secretaria-Executiva. O Plenário da Conitec realiza reuniões mensais, nas quais são avaliadas as demandas por incorporação, exclusão ou alteração das tecnologias no âmbito do SUS e atualização da Relação Nacional de Medicamentos Essenciais (Rename) ${ }^{5}$.

O suporte técnico e científico necessário para análise dessas demandas é exercido pela Secretaria-Executiva da comissão, que está sob responsabilidade do Departamento de Gestão e Incorporação de Tecnologias em Saúde (DGITS) da Secretaria de Ciência, Tecnologia e Insumos Estratégicos do Ministério da Saúde, auxiliado por uma rede de instituições nacionais (hospitais e universidades), parceiras da Conitec, que realizam estudos sob demanda do DGITS 5 .

O DGITIS foi criado pelo Decreto $\mathrm{n}^{0} 7.797$, de 30 de agosto de 2012, revogado pelo Decreto $\mathrm{n}^{0} 9.795 / 2019$ e alterado pelo Decreto $\mathrm{n}^{\circ}$ 9.816/2019. Possui as funções de acompanhar, subsidiar e dar suporte às atividades e demandas da Conitec e contribuir para a promoção do acesso e do uso racional de tecnologias seguras e eficientes, dentre outras. Esse departamento é composto pelas Coordenações: CoordenaçãoGeral de Gestão de Tecnologias na Saúde (CGGTS); Coordenação de Monitoramento e Avaliação de Tecnologias em Saúde (CMATS); Coordenação de Incorporação de Tecnologias (Citec); Coordenação de Gestão de Protocolos Clínicos e Diretrizes Terapêuticas (CPCDT) e Coordenação-Geral de Inovação Tecnológica na Saúde (CGITS) ${ }^{6}$.

As mudanças no processo de incorporação de tecnologias desde a criação da Conitec foram significativas, alterando a dinâmica e a qualidade da entrada de novos produtos no SUS. É necessário analisar seu perfil, pois são tecnologias ainda recentes, com potenciais impactos à saúde e envolvem recursos públicos necessários para disponibilizá-los aos cidadãos 1 .

A incorporação de tecnologias tem sido a principal responsável pelo aumento de custos dos sistemas nacionais de saúde, principalmente produtos industrializados, como os medicamentos. No SUS, o gasto anual com a compra de produtos e tecnologias para a saúde ultrapassa $\mathrm{R} \$ 20$ bilhões 7.

O aumento dos gastos públicos com produtos para a saúde deve-se, em parte, à 
incorporação formal de novas tecnologias ao SUS. E esta, consequentemente, pode estar associada ao fenômeno da judicialização da saúde. Entre 2010 e 2017, o Ministério da Saúde desembolsou $\mathrm{R} \$ 4,5$ bilhões para a compra de medicamentos, equipamentos, suplementos alimentares e cobertura de cirurgias e internações a partir de determinações judiciais. Em 2016, o Ministério da Saúde gastou $\mathrm{R} \$ 654,9$ milhões na compra de apenas 10 medicamentos, para atender a 1.213 pessoas $^{8}$.

O setor de saúde suplementar no Brasil difere em sua metodologia de incorporação de tecnologias. A revisão periódica do rol da ANS possui pouca relação com as diretrizes e orientações seguidas pela Conitec no processo de avaliação de tecnologias para o SUS, confirmando, dessa forma, a dicotomia existente entre os dois modelos. A utilização de um formulário de entrada de demandas, a solicitação de um parecer técnico-científico dos demandantes e a realização de consultas públicas pela ANS configuram as principais semelhanças entre os dois processos.

No entanto, a não divulgação dos relatórios de Avaliação de Tecnologias da Saúde, a falta da realização de avaliações econômicas e, principalmente, a pouca clareza dos critérios para recomendação da Agência, demonstram algumas das diferenças do processo da ANS em relação ao da Conitec ${ }^{9}$.

É essencial investigar o perfil das tecnologias incorporadas no SUS do Brasil, uma vez que, informações como essas são de relevância pública e servem como parâmetro para os gestores melhorarem a tomada de decisões, sanarem as deficiências relacionadas à incorporação de tecnologias em saúde e fazerem uso eficiente dos recursos.

O presente estudo teve como objetivo avaliar e descrever o perfil de tecnologias incorporadas pelo SUS, no período de janeiro de 2012 a setembro de 2019, além de investigar qual o demandante mais significativo e qual grupo de doenças é mais contemplada com a incorporação.

\section{Material e métodos}

Trata-se de um estudo exploratório, descritivo, retrospectivo, apresentando uma abordagem qualiquantitativa às demandas submetidas à Conitec, de $1^{\circ}$ de janeiro de 2012 a 30 de setembro de 2019. A coleta de dados foi realizada entre março e outubro de 2019. São dados secundários coletados por meio de análise documental, principalmente nas bases de dados disponíveis para consulta no site da Conitec (link: http:// conitec.gov.br/).

Foram considerados como tecnologias: (i) os medicamentos (medicamentos convencionais, vacinas, quimioterápicos e biológicos), (ii) procedimentos (procedimentos cirúrgicos, procedimentos assistenciais, exames de imagem, laboratoriais e outros), (iii) produtos (aparelhos, equipamentos e insumos utilizados na assistência à saúde) e (iv) Protocolos Clínicos e Diretrizes Terapêuticas (PCDTs) (protocolos clínicos e diretrizes terapêuticas, diretrizes diagnósticas e terapêuticas e protocolos de uso). Os PCDTs foram inclusos como tecnologias devido ao fato de diversos conceitos da literatura internacional e do próprio Ministério da Saúde assim o considerarem $\mathbf{1}$.

Para a análise estatística, foi utilizado, à priori, o Teste Qui-quadrado de Pearson, no caso em que os dados foram selecionados aleatoriamente, todas as frequências esperadas foram maiores ou igual a 1 e não mais que $20 \%$ das frequências esperadas foram inferiores a $5^{\mathbf{1 0}}$.

Nos casos em que foi verificada a impossibilidade deste, utilizou-se o Teste Exato de Fisher. Trata-se de um teste que calcula a probabilidade exata, mas que é limitado por não poder ser aplicado em casos em que os dados da amostra são muito grandes ${ }^{11}$.

A margem de erro utilizada na decisão dos testes estatísticos foi de $5,0 \%$. Os dados foram digitados em planilhas no Excel e o programa utilizado para obtenção dos cálculos estatísticos foi o IBM SPSS, na versão 23. 


\section{Resultados}

De acordo com a tabela 1, obteve-se um total de 380 tecnologias incorporadas, no período de 7 anos e 9 meses, com média de 49 incorporações por ano, sendo que 2018 também foi o ano com maior número de incorporação de tecnologias, perfazendo 76. Dentre essas 380 tecnologias incorporadas, 177 (46,6\%) eram medicamentos, $93(24,5 \%)$ eram procedimentos, 90 (23,7\%) eram PCDTs, e 20 (5,3\%) produtos.
Com relação aos demandantes, a demanda interna (secretarias, órgãos e instituições públicas das três esferas de governo vinculadas ao Ministério da Saúde) obteve 313 (82,4\%), externa (pessoas físicas e/ou jurídicas de direito privado) 58 (15,3\%) e interna e externa (quando a mesma tecnologia é solicitada por demandante interno e externo) 9 (2,4\%) do total de tecnologias incorporadas. Para a consulta pública, 281 (73,9\%) tecnologias foram submetidas a esse procedimento, enquanto 99 (26,1\%), não.

Tabela 1. Perfil das tecnologias incorporadas no SUS pela Conitec de 2012 a 2019

\begin{tabular}{|c|c|c|}
\hline Variável & $n$ & $\%$ \\
\hline TOTAL & 380 & 100,0 \\
\hline \multicolumn{3}{|c|}{ Ano da recomendação } \\
\hline 2012 & 33 & 8,7 \\
\hline 2013 & 27 & 7,1 \\
\hline 2014 & 54 & 14,2 \\
\hline 2015 & 55 & 14,5 \\
\hline 2016 & 30 & 7,9 \\
\hline 2017 & 64 & 16,8 \\
\hline 2018 & 76 & 20,0 \\
\hline 2019 & 41 & 10,8 \\
\hline \multicolumn{3}{|l|}{ Tipo de tecnologia } \\
\hline Medicação(a) & 177 & 46,6 \\
\hline Procedimento(b) & 93 & 24,5 \\
\hline PCDT(c) & 90 & 23,7 \\
\hline Produtos(d) & 20 & 5,3 \\
\hline \multicolumn{3}{|l|}{ Demanda } \\
\hline Interna(1) & 313 & 82,4 \\
\hline Externa(2) & 58 & 15,3 \\
\hline Interna e Externa(3) & 9 & 2,4 \\
\hline \multicolumn{3}{|l|}{ Consulta pública } \\
\hline Sim & 281 & 73,9 \\
\hline Não & 99 & 26,1 \\
\hline \multicolumn{3}{|c|}{ Fonte: Elaboração própria a partir de dados disponíveis no site da Conitec ${ }^{\mathbf{3}}$. } \\
\hline \multicolumn{3}{|c|}{$\begin{array}{l}\text { (a): medicamentos convencionais, vacinas, quimioterápicos e biológicos; (b): procedimentos cirúrgicos, procedimentos assistenciais, } \\
\text { exames de imagem, laboratoriais e outros; (c): protocolos clínicos e diretrizes terapêuticas, diretrizes diagnósticas e terapêuticas e } \\
\text { protocolos de uso; (d): aparelhos, equipamentos e insumos utilizados na assistência à saúde. (1): secretarias, órgãos e instituições públicas } \\
\text { das três esferas de governo vinculadas ao ministério da saúde; (2): pessoas físicas e/ou jurídicas de direito privado; (3): quando a mesma } \\
\text { tecnologia é solicitada por demandante(s) interno(s) e externo(s). }\end{array}$} \\
\hline
\end{tabular}


A tabela 2 analisa o tipo de tecnologia segundo o demandante. Obteve-se que os três tipos de demandantes conseguiram incorporar ao SUS mais medicamentos do que os demais tipos de tecnologias. Percentualmente, de todas as suas respectivas demandas incorporadas, os demandantes internos tiveram $37,4 \%$, externos $93,1 \%$ e interno e externo $66,7 \%$ para medicamentos. No entanto, em números absolutos, os demandantes internos foram superiores, com 117 incorporações. A associação entre as variáveis foi significativa $(\mathrm{p}<0,001)$.

Tabela 2. Avaliação do tipo de tecnologia segundo o demandante da Conitec entre 2012 a 2019

\begin{tabular}{|c|c|c|c|c|c|c|c|c|c|c|c|}
\hline \multirow[b]{3}{*}{ Demandante } & \multicolumn{11}{|c|}{ Tipo de tecnologia } \\
\hline & \multicolumn{2}{|c|}{ Medicamento } & \multicolumn{2}{|c|}{ Procedimento } & \multicolumn{2}{|c|}{ PCDT } & \multicolumn{2}{|c|}{ Produto } & \multicolumn{2}{|c|}{ Grupo Total } & \multirow[b]{2}{*}{ Valor de $\mathrm{p}$} \\
\hline & $\mathbf{n}$ & $\%$ & $\mathrm{n}$ & $\%$ & $\mathrm{n}$ & $\%$ & $n$ & $\%$ & $\mathrm{n}$ & $\%$ & \\
\hline Internoa & 117 & 37,4 & 91 & 29,1 & 88 & 28,1 & 17 & 5,4 & 313 & 100,0 & $\mathrm{p}^{(1)}<0,001^{*}$ \\
\hline Externob & 54 & 93,1 & 1 & 1,7 & - & - & 3 & 5,2 & 58 & 100,0 & \\
\hline Interno e externoc & 6 & 66,7 & 1 & 1,7 & 2 & 22,2 & - & - & 9 & 100,0 & \\
\hline TOTAL & 177 & 46,6 & 93 & 24,5 & 90 & 23,7 & 20 & 5,3 & 380 & 100,0 & \\
\hline
\end{tabular}

Fonte: Elaboração própria a partir de dados disponíveis no site da Conitec ${ }^{\mathbf{3}}$.

Internoa: secretarias, órgãos e instituições públicas das três esferas de governo vinculadas ao ministério da saúde; Externob: pessoas físicas e/ou jurídicas de direito privado; Interno e externoc: quando a mesma tecnologia é solicitada por demandante(s) interno(s) e externo(s):

*: Associação significativa ao nível de 5\%; (1): Através do teste Exato de Fisher.

A tabela 3 explana os demandantes com maior êxito na incorporação de tecnologias. Verificou-se que os órgãos públicos se sobressaíram em número de incorporações, principalmente as secretarias vinculadas ao Ministério da Saúde, como a Secretaria de Ciências Tecnologias e Insumos Estratégicos (128 tecnologias), a Secretaria de Atenção em Saúde (124 tecnologias) e Secretaria de Vigilância em Saúde (63 tecnologias).

A Associação Nacional de Grupos de Pacientes Reumáticos (Anapar) foi a única instituição do grupo de pacientes que teve suas demandas incorporadas, totalizando 06 tecnologias. Todas essas tecnologias são medicamentos biológicos utilizados no tratamento da artrite reumatoide, sendo quatro anticorpos monoclonais (Rituximabe, Tocilizumabe, Infliximabe e Adalimumabe) e duas proteínas de fusão (Abatacepte e Etanercept).

Ainda conforme a tabela 3 , várias empresas da indústria farmacêutica tiveram êxito em suas demandas. As três empresas que mais conseguiram incorporar tecnologias solicitadas foram Roche ${ }^{\circledR}$, GlaxoSmithKline ${ }^{\circledR} \mathrm{e}$ Novartis ${ }^{\circledR}$, com 05 tecnologias cada.

O poder judiciário também foi responsável por algumas tecnologias incorporadas. Sendo a $1^{\text {a }}$ Vara Federal de Porto Alegre responsável por 02 tecnologias, Justiça Federal da $4^{\mathrm{a}}$ Região - Seção Judiciária do Rio Grande do Sul por 01 tecnologia e $6^{\text {a }}$ Vara Federal de Sergipe por 01 tecnologia.

Ressalta-se que o somatório de tecnologias ultrapassa o número real de tecnologias incorporadas pelo fato de algumas tecnologias terem sido solicitadas por mais de um demandante. 
Tabela 3. Ranking de pessoas físicas ou jurídicas, de direito público ou privado, que tiveram êxito em incorporar tecnologias solicitadas entre 2012 a 2019

Demandante

Secretaria de Ciência, Tecnologia e Insumos Estratégicos/MS

Secretaria de Atenção à Saúde/MS

Secretaria de Vigilância em Saúde/MS

Agência Nacional de Vigilância Sanitária

Associação Nacional de Grupos de Pacientes Reumáticos - Anapar

Produtos Roche Químicos e Farmacêuticos S.A.

GlaxoSmithKline Brasil Ltda.

Novartis Biociências S.A.

AbbVie Farmacêutica Ltda.

Secretaria de Saúde do Estado de São Paulo

Janssen-Cilag Farmacêutica Ltda.

Laboratórios Pfizer Ltda.

Merck Sharp \& Dohme Farmacêutica Ltda.

Sociedade Brasileira de Diabetes - SBD

Sociedade Brasileira de Oncologia Clínica - SBOC

UCB BioPharma S/A

Secretaria Estadual de Saúde de Minas Gerais

1ㅡ. Vara Federal de Porto Alegre

Bristol-Myers Squibb Farmacêutica S.A.

AstraZeneca Brasil Ltda.

Biogen Brasil Produtos Farmacêuticos Ltda

BioMarin Brasil Farmacêutica

Gilead Sciences do Brasil Ltda.

Teva Farmacêutica Ltda.

Conselho Nacional de Secretários Municipais de Saúde - Conasems

Beaufour Ipsen Farmacêutica Ltda.

Biotronik Comercial Ltda.

Coloplast do Brasil

Justiça Federal da 4ำ Região - Seção judiciária do Rio Grande do Sul

LivaNova Brasil

Poder Judiciário Federal - Secão Judiciária de Sergipe - 6a Vara Federal

Sanofi-Genzyme

Sociedade Brasileira de Hemodinâmica e Cardiologia Intervencionista

Takeda

Zambon Laboratórios Farmacêuticos Ltda.

Gabinete do Ministro da Saúde

Instituto Nacional do Câncer - Inca

Prefeitura Municipal de Belo Horizonte-Hospital Risoleta Tolentino Neves

Secretaria Estadual de Saúde de Pernambuco

Secretaria Municipal de Saúde de Porto Alegre
№ de tecnologias incorporadas

128

124

63

14

6

5 
Tabela 3. (cont.)

\section{Demandante}

№ de tecnologias incorporadas

Ministério da Saúde

Instituto Nacional de Cardiologia

Sociedade Brasileira de Urologia

Laboratório Químico Bergamo Ltda.

Organização Pan-americana da Saúde

Universidade Federal de Minas Gerais - UFMG

Fonte: Elaboração própria a partir de dados disponíveis no site da $\mathrm{Conitec}^{\mathbf{3}}$

A tabela 4 demonstra que os medicamentos foram não só as tecnologias que mais passaram por consulta pública (44,1\%), mas também as que mais deixaram de se submeter a esse procedimento $(53,5 \%)$. A consulta pública é uma ferramenta importante para promover a democratização e transparência na escolha de tecnologias para o SUS, pois permite que a sociedade participe deste processo de escolha. No entanto, em algumas, como de extrema necessidade de alguns medicamentos, por exemplo, essas tecnologias são dispensadas da consulta pública.

Tabela 4. Tecnologias incorporadas que se submeteram à consulta pública pela Conitec entre 2012 e 2019

\begin{tabular}{lrrrrrrrrrrrrr}
\hline & & \multicolumn{1}{c}{ Tipo de tecnologia } \\
& Medicamento & Procedimento & \multicolumn{1}{c}{ PCDT } & \multicolumn{1}{c}{ Produto } & \multicolumn{2}{c}{ Grupo Total } \\
Consulta pública & $\mathbf{n}$ & $\%$ & $\mathbf{n}$ & $\%$ & $\mathbf{n}$ & $\%$ & $\mathbf{n}$ & $\%$ & $\mathbf{n}$ & $\%$ & Valor de p \\
\hline Sim & 124 & 44,1 & 50 & 17,8 & 88 & 31,3 & 19 & 6,8 & 281 & 100,0 & $P(1)<0,001^{\star}$ \\
Não & 53 & 53,5 & 43 & 43,4 & 2 & 2,0 & 1 & 1,0 & 99 & 100,0 & \\
TOTAL & 177 & 46,6 & 93 & 24,5 & 90 & 23,7 & 20 & 5,3 & 380 & 100,0 & \\
\hline
\end{tabular}

Fonte: Elaboração própria a partir de dados disponíveis no site da Conitec ${ }^{3}$

*Associação significativa ao nível de 5,0\%.

(1) Através do teste Qui-quadrado de Pearson.

De acordo com a tabela 5 , prevaleceu o grupo de 'algumas Doenças Infecciosas e Parasitárias (DIPs)', com 77 tecnologias, seguido de 'doenças endócrinas, nutricionais e metabólicas', com 42 tecnologias e, em terceiro, o grupo 'outros', com 39 tecnologias. 
Tabela 5. Tipo de tecnologia incorporada pela Conitec por categoria de doenças (CID-10)^ entre 2012 e 2019

\begin{tabular}{|c|c|c|c|c|c|}
\hline Tipo de tecnologia / categoria de doenças (CID-10) & Medicamentos & PCDT & Procedimentos & Produtos & Total \\
\hline Algumas doenças infecciosas e parasitárias (a00-b99) & 51 & 16 & 9 & 1 & 77 \\
\hline Neoplasias (c00-d48) & 15 & 10 & 8 & 3 & 36 \\
\hline $\begin{array}{l}\text { Doenças do sangue e dos órgãos hematopoéticos e alguns transtornos imunitá- } \\
\text { rios (d50-d89) }\end{array}$ & 4 & 5 & 2 & 1 & 12 \\
\hline Doenças endócrinas, nutricionais e metabólicas (e00-e90) & 20 & 16 & 4 & 2 & 42 \\
\hline Transtornos mentais e comportamentais (f00-f99) & 8 & 0 & 7 & 0 & 15 \\
\hline Doenças do sistema nervoso (g00-g99) & 12 & 6 & 2 & 0 & 20 \\
\hline Doenças do olho e anexos (h00-h59) & 1 & 4 & 3 & 0 & 8 \\
\hline Doenças do ouvido e da apófise mastóide (h60-h95) & 1 & 0 & 0 & 0 & 1 \\
\hline Doenças do aparelho circulatório (i00-i99) & 4 & 3 & 1 & 2 & 10 \\
\hline Doenças do aparelho respiratório (j00-j99) & 8 & 0 & 4 & 0 & 12 \\
\hline Doenças do aparelho digestivo (k00-k93) & 2 & 3 & 3 & 0 & 8 \\
\hline Doenças de pele e do tecido subcutâneo (100-199) & 10 & 2 & 0 & 0 & 12 \\
\hline Doenças do sistema osteomuscular e do tecido conjuntivo (m00-m99) & 23 & 9 & 0 & 0 & 32 \\
\hline Doenças do sistema geniturinário (n00-n99) & 2 & 1 & 1 & 1 & 5 \\
\hline Gravidez, parto e puerpério (000-০99) & 1 & 2 & 0 & 0 & 3 \\
\hline Algumas afecções originadas no período perinatal (p00-p96) & 0 & 0 & 0 & 0 & 0 \\
\hline Malformações congênitas, deformidades e anomalias cromossômicas (q00-q99) & 1 & 1 & 2 & 0 & 4 \\
\hline $\begin{array}{l}\text { Sintomas, sinais e achados anormais de exames clínicos e de laboratório, não } \\
\text { classificados em outra parte (r00-r99) }\end{array}$ & 0 & 0 & 0 & 4 & 4 \\
\hline $\begin{array}{l}\text { Lesões, envenenamento e algumas outras consequências de causas externas } \\
\text { (s00-t98) }\end{array}$ & 2 & 1 & 1 & 0 & 4 \\
\hline Causas externas de morbidade e de mortalidade (v01-y98) & 0 & 5 & 0 & 0 & 5 \\
\hline $\begin{array}{l}\text { Fatores que influenciam o estado de saúde e o contato com os serviços de saúde } \\
\text { (z00-z99) }\end{array}$ & 7 & 3 & 15 & 6 & 31 \\
\hline Códigos para propósitos especiais (u00-u99) & 0 & 0 & 0 & 0 & 0 \\
\hline Outros (doenças raras sem CID, múltiplas doenças e outras situações sem CID) & 5 & 3 & 31 & 0 & 39 \\
\hline TOTAL & 177 & 90 & 94 & 20 & 380 \\
\hline
\end{tabular}

Fonte: Elaboração própria a partir de dados disponíveis no site da Conitec $^{\mathbf{3}}$.

${ }^{*}$ ): Classificação Internacional de Doenças-10.

Dentre as DIPs mais beneficiadas, o HIV (Vírus da Imunodeficiência Humana) obteve maior número de incorporações voltadas para o seu tratamento, totalizando 19 tecnologias, correspondendo a $21,1 \%$ do total de tecnologias incorporadas para este grupo de doenças. Ressalta-se que a totalização de tecnologias por diagnóstico superou a quantidade de tecnologias para o grupo 'Algumas Doenças Infecciosas e Parasitárias' pelo fato de algumas tecnologias terem sido indicadas para mais de uma doença enquadrada neste grupo.

\section{Discussão}

De acordo com os dados do presente estudo, 2018 foi o ano com maior número de incorporações, mas não foi encontrado na literatura algo que justificasse esse maior número de tecnologias incorporadas. No entanto, os PCDTs (Material Complementar) tiveram uma incorporação massiva naquele ano, totalizando 33, inclusive, superaram o número de medicamentos incorporados, alavancando o número total de tecnologias incorporadas no 
referido ano. Esses documentos oficiais do SUS objetivam garantir melhor cuidado em saúde, já que são elaboradas com base em evidências científicas. São tecnologias que precisam ser atualizadas a cada dois anos ou quando houver inclusão, alteração ou exclusão de tecnologias provocando a necessidade de atualização dos cuidados em saúde ${ }^{\mathbf{2}}$.

O estudo evidenciou que os medicamentos foram o tipo de tecnologia mais incorporada. No estudo de Nunes e colaboradores ${ }^{\mathbf{1 3}}$ sobre incorporação de medicamentos pela Conitec, entre 2012 e 2015, foi constatado que os medicamentos já obtinham maior número de incorporações em relação aos demais tipos de tecnologias naquele período. Segundo os mesmos autores, tal fato não causa surpresa, pois a investigação e a inovação relacionada com este tipo de tecnologia também dominam o mercado e a comunidade científica.

No Brasil, pesquisas nessa área ainda são insuficientes, fazendo com que a comunidade científica nacional tenha que recorrer a pesquisas de outros países com maior expertise. Isso inviabiliza a produção local e encarece a aquisição de produtos para a saúde, já que há a necessidade de importação dos mesmos. Além disso, os países desenvolvidos costumam ter indústria farmacêutica local bastante desenvolvida, o que estimula a produção de pesquisa e desenvolvimento ${ }^{14}$.

Os demandantes internos tiveram superioridade em relação aos demais. O estudo de Caetano e colaboradores ${ }^{1}$ sobre a incorporação de novos medicamentos pela Conitec, entre 2012 e 2016, obtive números semelhantes ao do presente estudo. A demanda interna foi 77 (82,8\%), enquanto a externa foi de 16 (17,2\%). Apesar de ser um estudo voltado apenas para medicamentos, tal fato comprova a tendência de que órgãos e instituições vinculadas ao Ministério da Saúde sejam responsáveis por parte significativa das tecnologias incorporadas no SUS.

Essa superioridade se dá devido à nova política pública de gestão de tecnologias a partir da Conitec, o que conquistou a credibilidade das secretarias vinculadas ao Ministério da Saúde ${ }^{\mathbf{1 5}}$.

Todos os três tipos de demandantes conseguiram incorporar mais medicamentos do que os demais tipos de tecnologias, reforçando a importância que os demandantes dão para esse tipo de tecnologia. Além disso, os demandantes internos superaram em número de incorporação todos os outros demandantes, em todos os tipos de tecnologias.

Tal fato difere do que foi encontrado por Lima $^{16}$, que pesquisou as demandas da Conitec entre 2012 e 2014. Na época, tiveram prevalência as demandas da indústria, ou seja, demanda externa, nas tecnologias do tipo medicamentos e produtos. Portanto, este período avaliado no presente estudo, com predomínio dos demandantes internos, representa um aspecto positivo, tendo em vista que houve uma atenuação da influência das indústrias farmacêuticas, movidas pelo interesse econômico, frente ao mercado de consumo público ${ }^{16}$.

Diversos solicitantes externos tiveram êxito nas suas demandas. A exemplo da Anapar, que obteve mais sucesso, com quatro anticorpos monoclonais e duas proteínas de fusão utilizadas no tratamento da artrite reumatoide.

Isso se deve pelo fato de que medicamentos biológicos, como os anticorpos monoclonais e as proteínas de fusão, têm causado uma verdadeira revolução terapêutica em diversas áreas, dentre elas a reumatologia ${ }^{17}$.

A artrite reumatoide é uma doença inflamatória sistêmica que atinge, principalmente, as articulações, porém também pode afetar os pulmões, coração e outros órgãos. A estimativa é que esta doença acometa de 0,2 a $1 \%$ da população no Brasil. Isso representa 2 milhões de pessoas e, apesar da baixa prevalência, é uma doença que requer tratamento adequado por provocar limitações físicas aos pacientes ${ }^{\mathbf{1 8}}$.

A Anapar foi fundada em 26 de abril de 2006, na cidade do Rio de Janeiro (RJ). Ela tem como função proporcionar a integração de Grupos e Associações de Pacientes Reumáticos Brasileiros já constituídos, apoiar a criação de novos grupos, lutar pela defesa dos direitos 
dos pacientes e buscar por políticas públicas de saúde que permitam melhores condições de vida a esses pacientes ${ }^{19}$.

Dentre os demandantes externos, a indústria farmacêutica foi a mais prevalente, apesar de não ter tantas tecnologias incorporadas individualmente em relação aos demais grupos. A Roche ${ }^{\circledR}$, GlaxoSmithKline ${ }^{\circledR}$ e Novartis $^{\circledR}$ foram as que mais se destacaram, com 5 tecnologias cada, incluindo medicamentos, sendo proteínas de fusão, anticorpos monoclonais, antirretrovirais, vacinas antivirais, vasodilatadores, imunomoduladores e colinérgicos.

As três empresas são multinacionais de origem estrangeira. De acordo com dados do grupo Evaluate, que se trata de uma empresa líder em análise de mercado nos setores de biotecnologia e farmacêutico, a Roche ${ }^{\circledR}$ será líder no mercado de biológicos até 2022. A análise destaca que o laboratório tem o pipeline mais valorizado, ou seja, seu portifólio de produtos são mais aceitáveis pelos clientes e estima-se que alcance o patamar de $\mathrm{R} \$ 43$ bilhões de dólares em 2022. É provável que os investimentos da Roche ${ }^{\circledR}$ em pesquisa e desenvolvimento ultrapassem os da Novartis ${ }^{\circledR}$ e que os produtos daquela, em 2022 , representem $10 \%$ dos cinquenta produtos mais vendidos no mundo ${ }^{20}$.

Essa superioridade no número de demandantes da indústria farmacêutica entre os demandantes externos é atribuída à necessidade de infraestrutura e capacidade técnica para desenvolver estudos científicos, técnicos e econômicos, os quais são obrigatórios no processo de submissão da tecnologia junto à Conitec ${ }^{21}$.

Este estudo demonstrou que o poder judiciário, em menor número, também solicitou e teve suas demandas aceitas, o que leva a crer que os mesmos estão buscando soluções para o problema recorrente da judicialização de diversas tecnologias até então não disponibilizadas pelo SUS.

Nos últimos anos, o Ministério da Saúde gastou $\mathrm{R} \$ 4,5$ bilhões em medicamentos e outras tecnologias em saúde, cumprindo determinações judiciais. A incorporação de novas tecnologias pode estar sendo motivada por conta do fenômeno da judicialização como forma de melhorar a previsão de gastos em saúde 22 .

A judicialização na área da saúde não ocorre apenas no Brasil, mas também em países da América Latina e diversos outros países nos quais o direito à saúde é o fundamento legal de seus sistemas públicos de saúde. Com a instituição da Conitec, houve uma tentativa de modificar esse panorama. Ela disciplinou o processo de avaliação e incorporação de tecnologias em saúde no SUS, propiciando a tomada de decisão baseada em evidências, a transparência do processo administrativo de incorporação de tecnologias em saúde e o estabelecimento de mecanismos para a participação social23.

É rotina da Secretaria-Executiva da Conitec enviar informações e esclarecimentos técnicos a respeito de incorporação de tecnologias no SUS para diversos cidadãos, órgãos e instituições, proporcionando a interação com os Ministérios Públicos, a Advocacia Geral da União (AGU) e o Poder Judiciário. Essa atividade se traduz em contribuição, pois: (i) fornece esclarecimentos aos Procuradores dos Ministérios Públicos sobre a disponibilização de tecnologias em saúde pelo SUS, podendo evitar o ajuizamento de novas ações judiciais; (ii) fornece subsídios técnicos à AGU para a defesa da União em ações judiciais; e (iii) fornece subsídios técnicos aos juízes para que possam dispor de informações que lhes permitam tomar decisões em pedidos de concessão de liminares em ações judiciais ${ }^{24}$.

Um fato importante é que quase $74 \%$ do total de tecnologias passou por consulta pública. Isso representa um importante passo para fortalecer a participação social nos processos de incorporação de tecnologias no SUS, já que permite que a sociedade contribua com críticas, opiniões sobre a referida tecnologia, o que pode repercutir na aceitação ou não da mesma. Esse procedimento passou a ser obrigatório a partir da criação da Conitec, no ano de 2011.

O Decreto $n^{\circ} 7.646 / 2011$, que regulamentou os processos de avaliação de solicitações de incorporação no SUS, estabelece que todas as recomendações emitidas pelo Plenário sejam 
submetidas à consulta pública. Entretanto, o artigo 29 deste Decreto prevê a possibilidade de processo administrativo simplificado em casos de relevante interesse público, sem detalhar, contudo, em que situações isso se aplica $^{25}$. Isso justifica o fato de não haver consulta pública em todos os processos de avaliação de tecnologias pela Conitec.

Esta pesquisa demonstrou que a categoria de doenças mais beneficiada com incorporações foi 'Algumas Doenças Infecciosas e Parasitárias', com ênfase para o HIV. Parece ser paradoxal ao se buscar priorizar as doenças mais prevalentes no Brasil, que são as crônicas não transmissíveis, como hipertensão, diabetes, doença pulmonar obstrutiva crônica, asma e neoplasias. No entanto, as doenças infecciosas ainda são um problema de saúde pública no Brasil. Embora a proporção do total de mortes causadas por elas tenha caído de $50 \%$ para $5 \%$ ao longo dos últimos oitenta anos, essa redução tem sido mais pronunciada em algumas doenças infecciosas do que em outras ${ }^{26}$.

O Brasil enfrenta a persistência de doenças emergentes, como exemplo a mais importante introduzida na década de 70 , o HIV, que demanda a necessidade contínua de assistência à saúde e disponibilização de antirretrovirais de alta potência ${ }^{27}$. Portanto, é justificável o volume de tecnologias incorporadas para essa categoria, já que é necessário não só assegurar a erradicação de algumas doenças, mas também controlar o número de casos de doenças emergentes e reemergentes.

\section{Conclusões}

O processo de incorporação de tecnologias no SUS por meio da Conitec trouxe aspectos relevantes, principalmente a modernização, transparência e participação social. Tais fatores influenciam positivamente no resultado final. Houve um importante avanço na renovação de tecnologias incorporadas no SUS, principalmente medicamentos, através de um processo mais transparente.

Além disso, o estudo permitiu demonstrar a relevância dos medicamentos não só para a saúde pública no Brasil, mas também para os gastos públicos, os quais perfazem uma parcela importante das despesas. Compreender esses gastos torna-se importante para viabilizar a tomada de decisão de gestores, bem como direcionar, de forma equilibrada, as incorporações de tecnologias no SUS. Nesse contexto, sugere-se que investimentos na área de pesquisa e desenvolvimento de produtos para a saúde sejam estimulados para viabilizar a produção nacional e, consequentemente, reduzir os custos de aquisição pelo SUS e promover o acesso ao maior número possível de pacientes.

Conclui-se que o perfil das tecnologias incorporadas são principalmente medicamentos, por demanda interna, com indicação para DIPs e, sobretudo, para o HIV. Os medicamentos continuam sendo o foco das solicitações, no entanto as demandas internas passaram a ter mais espaço nesse cenário, acima de tudo as demandas provenientes de secretarias do Ministério da Saúde.

\section{Colaboradores}

Rodrigues Filho FJ (0000-0003-0886-1633)* contribuiu para a elaboração da pesquisa e artigo. Pereira MC (0000-0002-1672-8202)* contribuiu para a orientação da pesquisa e artigo. 


\section{Referências}

1. Caetano R, Silva RMD, Pedro EM, et al. Incorporação de novos medicamentos pela Comissão Nacional de Incorporação de Tecnologias do SUS, 2012 a junho de 2016. Ciênc. Saúde Colet. 2017; (22):2513-25.

2. Santos ZMSA, Frota MA, Martins ABT. Tecnologias em saúde: da abordagem teórica a construção e aplicação no cenário do cuidado. Fortaleza: EdUECE; 2016

3. Comissão Nacional de Incorporação de Tecnologias. Histórico institucional. [acesso em 2019 jul 9]. Disponível em: http://conitec.gov.br/historico-institucional.

4. Comissão Nacional de Incorporação de Tecnologias. Histórico institucional. [acesso em 2020 jan 12]. Disponível em http://conitec.gov.br/historico-institucional.

5. Rabelo RB, Petramale CA, Da Silveira LC, et al. A comissão nacional de incorporação de tecnologias no SUS: um balanço dos primeiros anos de atuação. Revista Eletrônica Gestão e Saúde. 2015; (4): 3225-3240.

6. Comissão Nacional de Incorporação de Tecnologias. [acesso em 2020 jan 12]. Disponível em: http://conitec.gov.br/entenda-a-conitec-2.

7. Guimarães R, Noronha J, Elias FTS, et al. Política de ciência, tecnologia e inovação em saúde. Ciênc. Saúde Colet. 2019; 24(3):881-886

8. Pierro B. Demandas crescentes: parcerias entre instituições de pesquisa e a esfera pública procuram entender a judicialização da saúde e propor estratégias para lidar com o fenômeno. Rev. Pesquisa Fapesp. 2017; 18(252):18-25.

9. Lisbôa R, Caetano R. Avaliação de Tecnologias em Saúde na saúde suplementar brasileira: revisão de escopo e análise documental. Saúde debate. 2021, 44:1255-1276.

10. Lima Filho MA. Teste de Independência. [acesso em
2020 jan 12]. Disponível em: http://www.de.ufpb. br/ luiz/AED/Aula10.pdf.

11. Campos GM. Estatística prática para docentes e pós-graduandos. São Paulo: Faculdade de Odontologia de Ribeirão Preto; 2001.

12. Brasil. Ministério da Saúde, Secretaria de Ciência, Tecnologia e Insumos Estratégicos. Protocolo Clínico e Diretrizes Terapêuticas do Diabete Melito Tipo 1. 2019. [acesso em 2020 fev 23]. Disponível em: http://conitec.gov.br/images/Relatorios/2019/Relatrio_PCDT-Diabetes-Mellitus-Tipo-1_2019.pdf.

13. Nunes LMN, Fonteles MMDF, Passos ACB, et al. Evaluation of demands of inclusion, exclusion and alteration of Technologies in the Brazilian Health System submitted to the National Committee on Technology Incorporation. Braz. J. Pharm. Sci. 2017; 53(2):1-12.

14. Petramale CA. Avaliação e incorporação: do quê precisamos realmente. OPAS/OMS-representação Brasil. Brasília, DF. 2016; 1(8).

15. Rabelo RB, Petramale CA, Silveira LC, et al. A comissão nacional de incorporação de tecnologias no SUS: um balanço dos primeiros anos de atuação. Rev. Eletr. Gest. Saúde. 2015; (4):3225-40.

16. Lima SGG. O processo de incorporação de tecnologias em oncologia no SUS: análise da Comissão Nacional de Incorporação de Tecnologias no Sistema Único de Saúde - Conitec. [dissertação]. Rio de Janeiro: Escola Nacional de Saúde Pública Sergio Arouca, Fundação Oswaldo Cruz; 2015.

17. IMS Health. Top 20 global products 2014. [acesso em 2019 nov 10]. Disponível em: http: //www.imshealth. com/files/web/Corporate/News/TopLine\%20Market\%20Data/2014/Top_20_Global_Products_2014. pdf.

18. Borssatto AGF. Incorporação de medicamentos imunobiológicos para artrite reumatoide no setor suplementar de saúde do Brasil: a percepção de pacientes, 
médicos e gestores. [dissertação]. São Paulo: Fundação Getúlio Vargas; 2019.

19. Biored Brasil. Associações que apoiam a Biored Brasil. 2016. [acesso em 2020 mar 3]. Disponível em https:// www.bioredbrasil.com.br/associacoes-que-apoiam-a-biored-brasil/.

20. Vidal TJ, Figueiredo TA, Pepe VLE. O mercado brasileiro de anticorpos monoclonais utilizados para o tratamento de câncer. Cad. Saúde Pública. 2018; (34):1-14.

21. Rego V. Eficiência no SUS como alternativa à judicialização das políticas públicas de saúde: a criação da Conitec e a utilização de triagem administrativa na concessão de medicamentos [monografia]. Ribeirão Preto: Universidade de São Paulo; 2013. [acesso em 2020 mar 3]. Disponível em: http://www.tcc.sc.usp. br/tce/disponiveis/89/890010/tce-20122013-094059/ publico/VictorRego.pdf.

22. Souza KAO, Souza LEPF, Lisboa ES. Ações judiciais e incorporação de medicamentos ao SUS: a atuação da Conitec. Saúde debate. 2018; (42)837-48.

23. Brasil. Lei $\mathrm{n}^{\circ} 12.401$, de 28 de abril de 2011. Altera a Lei 8.080, de 19 de setembro de 1990, para dispor sobre a assistência terapêutica e a incorporação de tecnologia em saúde no âmbito do Sistema Único de Saude - SUS. Diário Oficial da União. 29 Abr 2011. [acesso em 2020 mar 3]. Disponível em: https://www. jusbrasil.com.br/diarios/26364169/pg-1-secao-1-diario-oficial-da-uniao-dou-de-29-04-2011.

24. Simabuku EMG, Catanheide ID, D'agostino C, et al. Comissão nacional de incorporação de tecnologias no SUS e a judicialização do acesso à saúde. Revista Eletrônica Gestão e Saúde. 2015; (4)3024-3042.

25. Brasil. Decreto n ${ }^{0} 7.646$, de 21 de dezembro de 2011. Dispõem sobre a Comissão Nacional de Incorporação de tecnologias no Sistema Único de Saúde e sobre o processo administrativo para incorporação, exclusão e alteração de tecnologias em saúde pelo Sistema Único de Saúde, e dá outras providências. Diário Oficial da União. 21 Dez 2011.

26. Barreto ML, Teixeira MG, Bastos FI, et al. Saúde no Brasil. Sucessos e fracassos no controle de doenças infecciosas no Brasil: o contexto social e ambiental, políticas, intervenções e necessidades de pesquisa. Lancet. 2015; 377(9780):47-60.

27. Waldman EA, Sato APS. Trajetória das doenças infecciosas no Brasil nos últimos 50 anos: um contínuo desafio. Rev. Saúde Públ. 2016; (50):68.

\footnotetext{
Recebido em 17/09/2020

Aprovado em 28/07/2021

Conflito de interesses: inexistente

Suporte financeiro: não houve
} 